\title{
Human Capital and its Demand in the Mining Industry
}

\author{
Olga Zaplatina ${ }^{1, *}$, and Rashit Bikmetov ${ }^{1}$ \\ ${ }^{1}$ T.F. Gorbachev Kuzbass State Technical University, 650000, 28 Vesennyaya St., Kemerovo, Russia
}

\begin{abstract}
The article highlights the problem of the importance of human capital for mining enterprises. The author substantiates the increase of the importance of the health capital of an individual as a determining component of human capital. The results of professional studies in the field of mining are presented, which allow us to ascertain the high demand for such important professional qualities as neuropsychic stability, physical and mental health. The article provides a definition of the health capital, its direct relationship with the quality of vocational training and the level of formation of professionally important qualities of future specialists in the mining industry. The author analyzes the problems of improving the quality of vocational training and increasing the health capital in the context of educational and extracurricular activities related to physical training and the education of professionally important psychophysiological qualities. The results of the study confirm the need for physical culture and health and sports activities for increasing the capital of health and managing the psychoemotional state with the purpose of qualitative preparation for the performance of professional activities in conditions of increased extremity.
\end{abstract}

\section{Introduction}

The current socio-economic situation and its trends in the development of mining require a special approach to the management and humane use of human resources. The presence of highly qualified personnel is the main factor in the long-term development of mining industrial production. Managing human capital through awareness of the escalation of its role is of great interest both in the scientific plan and in terms of the possibility of solving a number of problems in the mining industry.

To date, providing the mineral-raw complex with only the highest level of professional training is not enough. On the part of employers, there has recently been an increasing demand for professionals with a high level of health, working capacity, physical and psychophysiological capabilities [1].

Nevertheless, the health capital as the most important component of human capital is not assessed as determining social and industrial value, which can become a decisive factor in increasing the productive capacity of enterprises [2].

\footnotetext{
* Corresponding author:: zaplatinaoa@mail.ru
} 
The capital of health, preserved and multiplied as a social value and an inalienable need for production relations, will fully manifest itself in man and other elements of human capital. Namely:

-level of education, including knowledge, skills, skills and competences (including general, professional and special);

-professional-applied preparedness (skill level, work experience in production);

-level of economically significant awareness, since human capital itself is understood as the totality of the qualities of a person, his abilities to work, which determine his productivity and can become a source of income for the person, organization and society as a whole, because the person himself (according to T. Schulz) and its production capabilities "above all other forms of wealth combined";

-capital of migration, which provides professional mobility of employees;

-motivation of labor activity, closely knitted with the values of human values.

Human capital, characterizing the above human capabilities, will be expressed in its social potential.

Since scientific and technological progress in the mining industry is accompanied by a continuous increase in the role of human capital in ensuring high efficiency and safety of work, there is an urgent need to study and form those necessary mental and physical qualities of a specialist that will be in demand in the conditions of their future professional activity. Despite the progressive decline in gross muscular effort in modern mining, the success of professional work, working capacity continues to be conditioned by psychophysical capacity, mental stability, based, in addition to personal qualities, at the level of the physical condition of a specialist.

From the point of view of domestic representatives of engineering psychology, such scientists as VA Bodrov and AI Galaktionov, the level of development of neuropsychic resistance and anxiety plays the most significant role in the performance of professional activity in production conditions characterized by a certain degree of extremism [3]. In the plan of prevention and diagnosis of emotional state and mental health, special importance, according to AI Galaktionov, is given to the neuropsychic stability of the individual, which is an indicator of both mental and physical health of a person. Neuropsychic resistance shows the risk of maladaptation of a person in critical conditions caused by external or internal factors.

\section{Methods and Organization of the Study}

The research used a set of theoretical and empirical methods (theoretical analysis of psychological and pedagogical literature, questionnaires, questionnaires, pedagogical observations, pedagogical experiments), which make it possible to assess and improve the level of the neuropsychological stability of students at a mining institute in the process of physical education. The study of neuropsychological stability was carried out using the Forecast method developed by the St. Petersburg Military Medical Academy, which is informative in selecting specialists for work in difficult, unpredictable conditions, where increased demands are placed on the individual. The reliability of the results of the study was confirmed with the help of methods of mathematical statistics: the calculation of the mean value, the standard deviation; calculation of the Student's ratio, Fisher coefficient, comparison of variances; detection of regression dependence.

576 students of the day department of the Kuzbass State Technical University, 33 students of the correspondence department of the Kuzbass State Technical University who have worked in the production conditions from 5 to 10 years, 162 students of the engineering department of the Kemerovo State Agricultural Institute and 185 students of 
the Kemerovo State University at the Faculty of Chemistry and the Faculty of Physical Culture and Sports.

\section{Results and Discussion}

Analyzing the initial indices of neuropsychological stability (NPS), we see that the majority of students, both boys and girls, have a low level characterizing this quality of personality (Fig. 1).

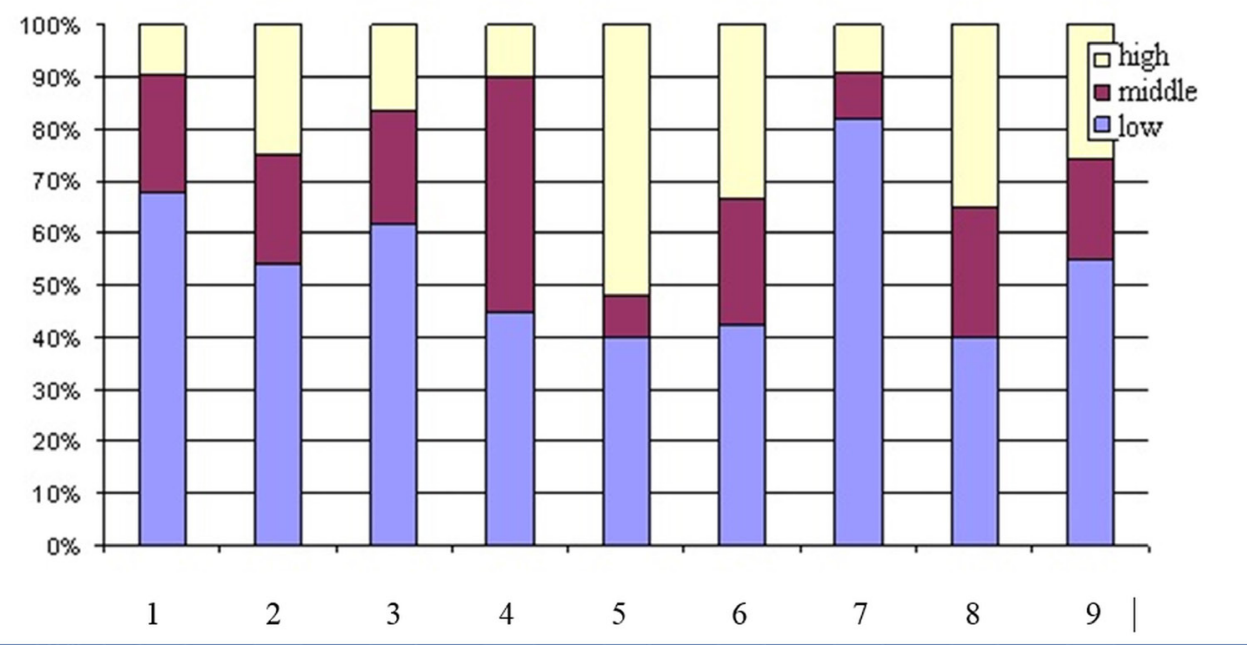

Fig. 1. The results of statistical observations in the study of neuropsychic resistance (NPS) in students of a technical university at the stating stage of the experiment, $\%$.

1, 2, 3 - indicators of students of the Mining Institute (girls, boys and regardless of sex, 4-6 indicators of students of the Institute of Chemical and Oil and Gas Technologies (for comparison) and 7-9 - indicators of students of the construction institute, respectively (for comparison)

So the students of the Mining Institute are dominated by a low level of neuropsychic resistance $(61.8 \%$ at the institute, $67.7 \%$ in girls and $54.2 \%$ in young men). This indicates the low stress-resistance of students.

A different picture is observed among the young men of the Institute of Chemical and Oil and Gas Technologies: here the high level of neuropsychic resistance was $52.0 \%$, exceeding the low by $12.0 \%$, and the average at $44.0 \%$; However, a survey and analysis of the documentation showed that $80.0 \%$ of the surveyed young men of this institute are those who go in for sports outside of school hours, attend sports sections, electives, additional classes. The students of the construction institute are dominated by a low level of neuropsychic resistance $(54.8 \%$ in general at the institute, $81.8 \%$ in girls and $40.0 \%$ in boys).

On the basis of the results of the ascertaining experiment, we concluded that the students enrolled in the first year of higher education characterize, basically, a low level of neuropsychic resistance, which, in our opinion, is associated with new living conditions for them, the nature of the training work, the low level of adaptation in an unfamiliar environment for a given period of time. This can also be characterized by high personal anxiety of students, their individual mental qualities.

As you know, physical training and sports, competitive activities help to build and improve the mental qualities "necessary to successfully confront downtrodden factors (uncertainty in their abilities, fear, stiffness, etc.), and also contribute to the increase of the level of neuropsychological stability, reducing susceptibility to a stressful situation [4]. In 
this regard, in the course of physical training of students of a technical college, along with in-depth differentiation and an individual approach, we used a variety of techniques in teaching and training, to improve the level of mental stability unequally students. For students with different levels of formation of the above-mentioned professionally important qualities, their specific tasks were formulated that contribute to maintaining or improving the level of the formation of data of professionally important qualities. Considered the types of physical culture and sports activities for these students, based on material and technical support of the process of physical education; the methods of teaching and training work that contribute to the positive influence on the formation of professionally important qualities and mental health of the future representative of the mining industry were considered.

For students with a high level of neuropsychological stability, inclusion in competitive activities was practiced; Instruction of organizational activities for the planning of individual parts of the training process; use of individual training programs; training in complex coordination exercises; Showing the performance in front of a group of a certain part or the whole exercise, demonstrating the technique of doing the exercise; attachment for training less-trained university students; appointment to more difficult stages of the relay race, obstacle course; involvement in the participation and organization of demonstration speeches; involvement in sectional work; staffing the team in gaming activities in such a way that students with a high level of stress resistance were in different teams (to balance the strength of teams and the ability to show themselves to other students); independent recruitment of teams; games with different in terms of training partners.

For students with an average level of neuropsychological stability, we used the following techniques: they offered more complex exercises for performance; used more diverse relay races; changed or established various rules of the game; in game situations, assign students' data to the role of players performing more complex actions or bearing greater responsibility for the outcome of the game; used multiple, close monitoring of the performance of exercises; included these students in a stronger team or formed game pairs (for example, tennis) with a stronger partner; appointed as leading in the performance of various exercises (both in motion and in static); They offered these students to demonstrate general development exercises in the process of various parts of the lesson.

For students with a low level of neuropsychological stability, the following approaches were used: involvement in competitive activities; more frequent use in the lessons of game situations, relay races, components of competitive activity; captains of teams; during the competitions, students were divided into subgroups according to the levels of training in order that they had the opportunity at that level of professionally important physical and mental qualities to occupy the leading and prizes; involved in organizing activities for the implementation of collective campaigns, charged with responsible actions; held constant control in physical culture and sports, constantly showed interest and attention to such students, used stimulating methods (praise, approval); appointed as leaders, captains of teams, "attackers" in the game; gave various recommendations for the implementation of exercises, interest and encouraging students; more often noticed positive changes in the level of training, complicating tasks; gave students an opportunity to feel the importance and necessity of their actions in collective activities.

Depending on the planned training and training work, the possibilities of its influence on the health and functional state of the body of university students, the formation and improvement of professionally important physical and mental qualities were considered. Thus, the training and training activities, organized with the application of the above methods of work, contributed to the improvement of the professionally important qualities of students, the impact on improving their level of preparation for future professional activities. 
After the completion of the formative experiment, the level of neuropsychic resistance (NPS) among students of the institutions studied has changed. So the number of girls from the Mining Institute with a high level of NPS increased by $16.1 \%$, with the average - by $22.6 \%$ and became predominant; in young men the high-level indicator became predominant and increased by $37.5 \%(\mathrm{p}<0.05)$. Girls of the Institute of Chemical and Oil and Gas Technologies showed a high level of NPS increased by $5.0 \%$ and exceeded the low level by $15.0 \%$; the number of students with an average level was $55.0 \%$. In young men, the high level of neuropsychological stability maintained its rank position and increased by $16.0 \%(p>0.05)$. Among the girls of the construction institute, there was an increase in the number of students with a high level of NPS and amounted to $18.2 \%$, exceeding the former by $9.1 \%$; a low level of $54.5 \%$, and an average of $27.3 \%$, an increase of $18.2 \%$. Among the young men of this institute, the number of students with a low level of NPS decreased by $15.0 \%$, and with the average level increased by $15.0 \%$; the number of students with a high level was preserved and amounted to $35.0 \%$ ( $>>0.05$ ) (Fig. 2).

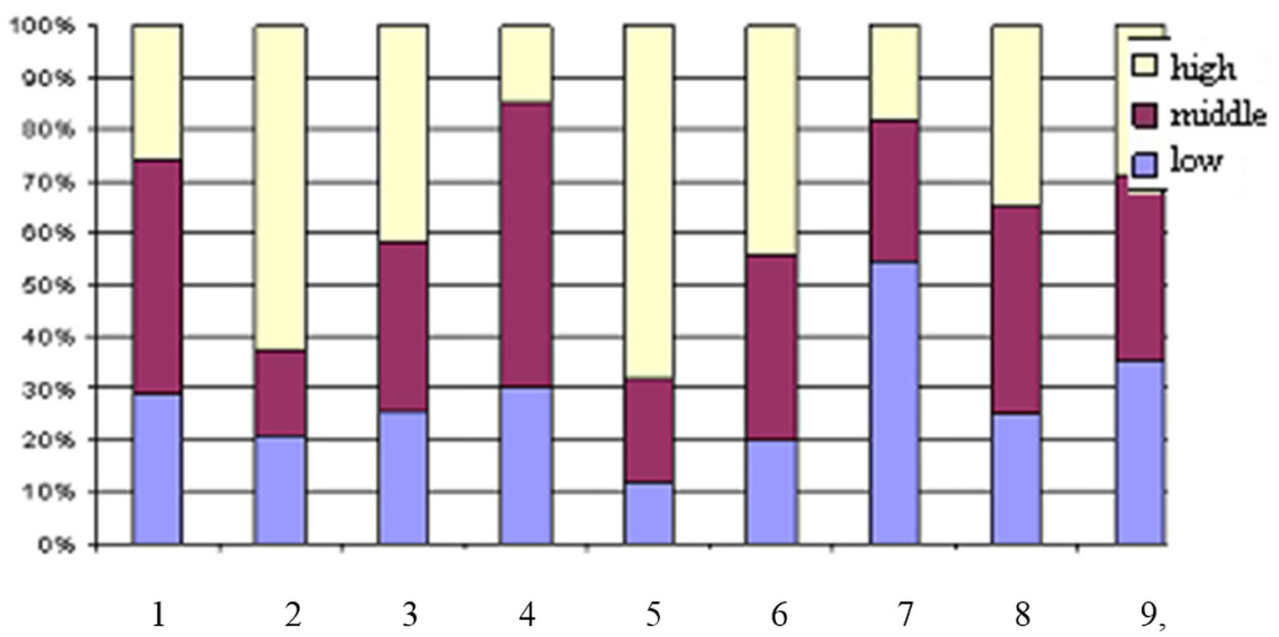

Fig. 2. The results of statistical observations in the study of neuropsychic resistance (NPS) in students of the technical university at the formative stage of the experiment, $\%$

1, 2, 3 - indicators of students of the Mining Institute (girls, boys and regardless of sex, 4-6 -

indicators of students of the Institute of Chemical and Oil and Gas Technologies (for comparison) and 7-9 - indicators of students of the construction institute, respectively (for comparison).

In order to convince ourselves of the legitimacy of the foregoing assertion that physical fitness and competitive activity helps us learn to resist the "knocking out" factors of the external environment, we undertook a comparison of the indices of neuropsychological stability of the students of the technical university, obtained after the pedagogical experiment with the indices of the students of the classical university, faculty "Physical Culture and Sport" and again to compare the data of the group of indicators with the results of the research students of correspondence department - workers of mines, sections, enterprises of mining industry. It turned out that the students of the third year of the faculty "Physical Culture and Sport" indicators of low and medium levels of neuropsychological stability are equal and noted in $42.9 \%$ of the respondents; the indicator of a high level of neuropsychic resistance is $14.2 \%$. In the students of the correspondence department working in mines and sections, the average indicator of neuropsychological stability prevails and amounted to $45.5 \%$, exceeding the high level by only $9.2 \%$; the indicator of low neuropsychological stability is rather small and amounted to only $18.2 \%$. 
Comparing the obtained results of the NPS with the indicators of the students of the correspondence department of the Kuzbass State Technical University working in production, we note the tendency to a similarity in the prevalence of high and medium levels of neuropsychic resistance; when assessing the same NPS in students of other universities we see that their results are somewhat worse than those of students of the Kuzbass State Technical University (Fig. 3).

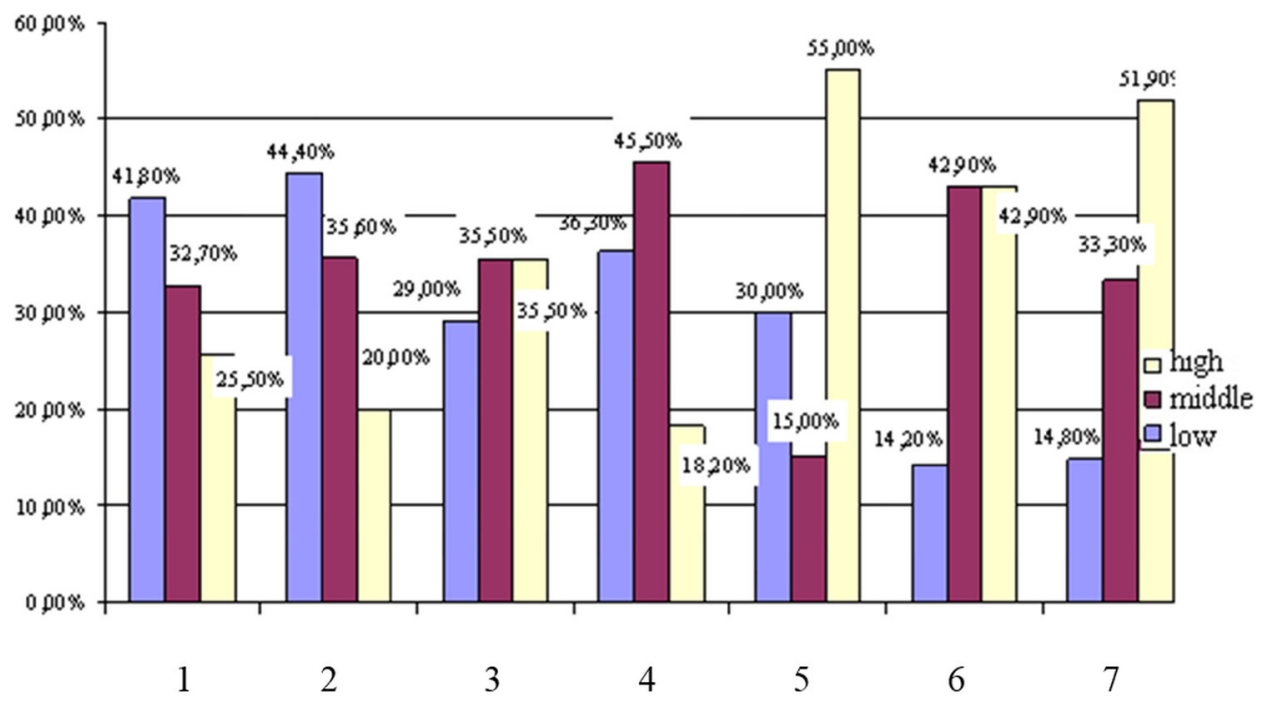

Fig. 3. Distribution of students of various universities and specialties according to levels of neuropsychic stability, \%

1 - students of the Mining Institute, 2 - students of the Institute of Chemical and Oil and Gas Technologies, 3 - students of the Construction Institute, 4 - employees of mining enterprises, 5 students of the classical university, 6 - athletes, 7 - students of the Faculty of Engineering of the Agricultural Institute.

Thus, we can conclude that the physical education of students of a technical university, the organization of this educational and educational process aimed at the formation and improvement of professionally important qualities plays an essential role in the formation of the personality of the future specialist, positively influencing the strengthening of mental health, the formation of mental qualities of the individual, contributing to the increase of the human capital of the mining industry.

Proceeding from the fact that the level of anxiety, neuropsychological stability, on which the behavior of a person depends in a stressful situation, is one of the most important components of a specialist's health capital, and also one of the basic requirements for employers in the professions of interest to us, we decided to turn to quality data special attention $[3 ; 5 ; 6 ; 7 ; 8]$.

In the process of completing the pedagogical experiment, we found a regression dependence in the change in the level of neuropsychiatric (NPS) stability of students, the institutions we studied. The sample was made up of students who, in our view, are characterized by a high level of preparation for professional activity, that is, with a steady motivation to increase the health potential for successful preparation for future work in production conditions $(\mathrm{n}=131)$.

This relationship allowed us to determine how many years students will need in order to attain the level of the attitude towards physical education formed at the end of the experiment as a factor in preparation for future professional activity, to achieve the level of 
emotional stability that prevails among workers in mining enterprises with experience in production conditions from 5 to 10 years.

As a result of this analysis, it was established that the students of the Mining Institute will need 2.8 years from the beginning of studies at the university, the Institute of Chemical and Oil and Gas Technologies - 1.08, and the Construction Institute - 2.5 years.

\section{Conclusion}

Based on the above results, we can conclude that the time allotted to physical education in the framework of the curriculum of students of a technical university is certainly insufficient to fully implement successful preparation for the forthcoming professional activity in the conditions of mining, however, in the current situation You can achieve increased effectiveness in the formation and improvement of professionally important personal qualities that ensure the growth of human resources capital within the capital of health.

So, the construction of the content of the educational and educational process of physical education for the purpose of improving the health capital contributes to the formation of students' conscious attitude to physical self-education, the formation of the need for psychological and functional self-diagnosis, as well as their awareness that the improvement of these qualities is a condition for qualitative preparation for the future professional activities and ensuring a sustainable development strategy for the mining industry.

\section{References}

1. R. A. Tsaturyan, Mining Information and Analytical Bulletin, 12, 438 (2009)

2. V. I. Kozlov, N. V. Povednyuk, Safety of enterprises' life in the industrially developed regions (KuzsTU, Kemerovo, 2011)

3. D. N. Gavrilov, The Theory and Practice of Physical Culture, 3, 9 (2003)

4. A. I. Galaktionov, Psychological Journal, 3, 14 (1992)

5. V. S. Ezhkov Vocational-applied training of students of machine-building specialties on the basis of basic psychophysiological properties of the individual (Nauka, Moscow, 2003)

6. V. M. Naskalov, Vocational training of students in the specialty "Chemical production (Belarus, Minsk, 1991)

7. Zh. K. Kholodov, Theory and Methods of Physical Education and Sport (Nauka, Moscow, 2001)

8. V. K. Shemanaev, Vocational training of students in mine surveying and geodetic specialties (Nauka, Moscow, 1991) 